\title{
On some new weakly singular Volterra integral inequalities with maxima and their applications
}

Yong Yan $^{*}$

\section{"Correspondence:} kdyan698@163.com Department of Mathematics, Sichuan University for Nationalities, Kangding, Sichuan 626001, P.R. China

\begin{abstract}
In this paper, we consider a general form of nonlinear integral inequalities with the unknown function composed with a given function on the left hand side, more than one distinct nonlinear integrals on its right-hand side, and weakly singular kernels, and involving maxima of unknown function. Requiring neither monotonicity nor separability of given functions, we apply monotonization to estimate the unknown function. Our result can be used to weaken conditions for some known results. We apply the obtained result to a boundary value problem of integro-differential equations with maxima for uniqueness.
\end{abstract}

MSC: 26D10;26D15

Keywords: integral inequalities; weakly singular; integro-differential equations with maxima; uniqueness

\section{Introduction}

The Gronwall-Bellman inequality $[1,2]$ is an important tool in the study of existence, uniqueness, boundedness, stability, invariant manifolds, and other qualitative properties of solutions of differential equations and integral equations. There can be found a lot of its generalizations in various cases in the literature (e.g. [3-6]). Lipovan [7] investigated the retarded integral inequality

$$
u(t) \leq c+\int_{t_{0}}^{t} f(s) \omega(u(s)) d s+\int_{\alpha\left(t_{0}\right)}^{\alpha(t)} g(s) \omega(u(s)) d s, \quad t_{0} \leq t<t_{1} .
$$

Their results were further generalized by Agarwal et al. [8] in 2005 to the inequality

$$
u(t) \leq a(t)+\sum_{i=1}^{n} \int_{\alpha_{i}\left(t_{0}\right)}^{\alpha_{i}(t)} f_{i}(t, s) \omega_{i}(u(s)) d s, \quad t_{0} \leq t<t_{1}
$$

Another aspect of integral inequalities is to consider the unknown $u$ composed with a given function on the left hand side, which has been developed (see [9-13]). On the basis of discussion (see [14-16]) on integral inequalities in multi-variables.

(c) 2015 Yan. This article is distributed under the terms of the Creative Commons Attribution 4.0 International License (http://creativecommons.org/licenses/by/4.0/), which permits unrestricted use, distribution, and reproduction in any medium, provided you give appropriate credit to the original author(s) and the source, provide a link to the Creative Commons license, and indicate if changes were made. 
In recent years, many researchers have devoted many efforts to investigating weakly singular integral inequalities and their applications (see [17-22]). In 1979 McKee [17] considered the following initial value problem:

$$
y^{\prime}(t)=f(t, y)+c \int_{0}^{t} \frac{y^{\prime}(s)}{(t-s)^{\alpha}} d s+q(t), \quad 0 \leq t \leq T, \quad y(0)=y_{0},
$$

when $\alpha=\frac{1}{2}$ for the diffusion of discrete particle in a turbulent fluid. Henry [18] used integral inequality with singular kernel to prove global existence and exponential decay results for a parabolic differential equation. Medved [19] presented a new method to discuss nonlinear singular integral inequalities of Henry type

$$
u(t) \leq a(t)+b(t) \int_{t_{0}}^{t}(t-s)^{\beta-1} s^{\gamma-1} F(s) u(s) d s, \quad t \geq 0
$$

In 2008 Ma and Pečairé [21] considered the following nonlinear singular inequalities with power nonlinearity:

$$
u^{p}(t) \leq a(t)+b(t) \int_{t_{0}}^{t}\left(t^{\alpha}-s^{\alpha}\right)^{\beta-1} s^{\gamma-1} f(s) u^{q}(s) d s, \quad t \geq 0 .
$$

Along with the development of automatic control theory and its applications to computational mathematics and modeling, attention was also put to integral inequalities with the maxima of the unknown function. Actually, many problems in the control theory can be modeled in the form of differential equations with the maxima of the unknown function $[23,24]$. For example, the equation describing the work of the regulator [25] can be presented as

$$
T_{0} u^{\prime}(t)+u(t)+q \max _{s \in[t-h, t]} u(s)=f(t)
$$

where $T_{0}$ and $q$ are constants. Equations involving maxima of an unknown function are called differential equations with maxima $[23,24]$. Such a problem again requires a new type of integral inequalities as a tool to investigate its qualitative properties. There have been given some results for integral inequalities containing the maxima of the unknown function [26-29].

In 2014 Thiramanus et al. [30] considered the following system of integral inequalities:

$$
\begin{aligned}
& u(t) \leq r(t)+\int_{t_{0}}^{t}(t-s)^{\alpha-1}\left[p(s) u(s)+q(s) \max _{\xi \in[\beta s, s]} u(\xi)\right] d s, \quad t \in\left[t_{0}, T\right), \\
& u(t) \leq \phi(t), \quad t \in\left[\beta t_{0}, t_{0}\right]
\end{aligned}
$$

where $\alpha>0,0<\beta<1, r, p, q$, and $\phi$ are nonnegative continuous functions.

In this paper we generally consider the system of integral inequalities

$$
\begin{aligned}
\varphi(u(t)) \leq & a(t)+\sum_{i=1}^{m} \int_{b_{i}\left(t_{0}\right)}^{b_{i}(t)}\left(t^{\alpha_{i}}-s^{\alpha_{i}}\right)^{k_{i}\left(\beta_{i}-1\right)} s^{q_{i}\left(\gamma_{i}-1\right)} g_{i}(t, s) \omega_{i}(u(s)) d s \\
& +\sum_{j=m+1}^{m+n} \int_{b_{j}\left(t_{0}\right)}^{b_{j}(t)}\left(t^{\alpha_{j}}-s^{\alpha_{j}}\right)^{k_{j}\left(\beta_{j}-1\right)} s^{q_{j}\left(\gamma_{j}-1\right)} g_{j}(t, s)
\end{aligned}
$$




$$
\begin{gathered}
\times \omega_{j}\left(\max _{\xi \in\left[c_{j}(s)-h, c_{j}(s)\right]} f(u(\xi))\right) d s, \quad t \in\left[t_{0}, t_{1}\right), \\
u(t) \leq \psi(t), \quad t \in\left[b^{*}\left(t_{0}\right)-h, t_{0}\right],
\end{gathered}
$$

where $a, f, g_{i}$ 's, and $\omega_{i}$ 's are nonnegative continuous functions, $b_{i}$ 's are nonnegative continuously differentiable and nondecreasing functions and $b^{*}\left(t_{0}\right):=\min \left\{\min _{1 \leq i \leq m} b_{i}\left(t_{0}\right)\right.$, $\left.\min _{m+1 \leq j \leq m} c_{j}\left(b_{j}\left(t_{0}\right)\right)\right\}$. As required in previous work [26, 27], we suppose that $0 \leq b_{i}(t) \leq t$, $h>0$, is a constant and the $\omega_{i}$ 's are definite positive, i.e., $\omega_{i}(s)>0$ for $s>0$. In this paper we require neither monotonicity of $a, \omega_{i}$ 's, $g_{i}$ 's and $g$ nor $a(t) \geq 1$. We monotonize those $\omega_{i}$ 's to make a sequence of functions in which each possesses stronger monotonicity than the previous one so as to give an estimation for the unknown function. Finally, we apply the obtained result to a boundary value problem of integro-differential equations with maxima for uniqueness.

\section{Main result}

Consider system (1.6) of integral inequalities with $t_{0}<t_{1}$ in $\mathbb{R}_{+}:=[0, \infty) . C(M, S)$ denotes the class of all continuous functions defined on set $M$ with range in the set $S . B(\xi, \eta)=$ $\int_{0}^{1} s^{\xi-1}(1-s)^{\eta-1} d s(\xi, \eta \in \mathbb{C}, \operatorname{Re} \xi>0, \operatorname{Re} \eta>0)$ is the well-known beta function. As in [9], we say $\mu_{1} \propto \mu_{2}$ for $\mu_{1}, \mu_{2}: A \subset \mathbb{R} \rightarrow \mathbb{R} \backslash\{0\}$ if $\mu_{2}(s) / \mu_{1}(s)$ is nondecreasing on $A$.

Suppose that

(H1) all $b_{i}:\left[t_{0}, t_{1}\right) \rightarrow \mathbb{R}_{+}(i=1,2, \ldots, m+n), c_{j}:\left[t_{0}, t_{1}\right) \rightarrow \mathbb{R}_{+}(j=m+1, m+2, \ldots, m+n)$ are continuously differentiable and nondecreasing such that $b_{i}(t) \leq t, c_{j}(t) \leq t$ on $\left[t_{0}, t_{1}\right)$

(H2) $f, \varphi: \mathbb{R}_{+} \rightarrow \mathbb{R}_{+}$, and $\psi:\left[b^{*}\left(t_{0}\right)-h, t_{0}\right] \rightarrow \mathbb{R}_{+}$are continuous functions, $\varphi$ is strictly increasing such that $\lim _{t \rightarrow+\infty} \varphi(t)=+\infty$;

(H3) all $g_{i}(t, s)(i=1,2, \ldots, m+n)$ are continuous and nonnegative functions on $\left[t_{0}, t_{1}\right) \times\left[b^{*}\left(t_{0}\right), t_{1}\right)$

(H4) all $\omega_{i}(i=1,2, \ldots, m+n)$ are continuous on $\mathbb{R}_{+}$and positive on $(0,+\infty)$;

(H5) $a(t)$ is continuous and nonnegative function on $\left[t_{0}, t_{1}\right)$;

(H6) $k_{i}, q_{i} \in[0,1], \alpha_{i} \in(0,1], \beta_{i} \in(0,1), p q_{i}\left(\gamma_{i}-1\right)+1>0, p k_{i}\left(\beta_{i}-1\right)+1>0$ such that $\frac{1}{p}+k_{i} \alpha_{i}\left(\beta_{i}-1\right)+q_{i}\left(\gamma_{i}-1\right) \geq 0(p>1, i=1,2, \ldots, m+n)$.

For those $\omega_{i}^{\prime}$ 's given in (H4), define $\tilde{\omega}_{i}(t)$ inductively by

$$
\left\{\begin{array}{l}
\tilde{\omega}_{1}(t):=\max _{\tau \in[0, t]}\left\{\bar{\omega}_{1}(\tau)\right\}, \quad t \geq 0, \\
\tilde{\omega}_{i+1}(t):=\max _{\tau \in[0, t]}\left\{\frac{\bar{\omega}_{i+1}(\tau)}{\tilde{\omega}_{i}(\tau)}\right\} \tilde{\omega}_{i}(t), \quad t \geq 0, i=1,2, \ldots, m-1, \\
\tilde{\omega}_{m+1}(t):=\max _{\tau \in[0, t]}\left\{\frac{\left.\left\{\hat{\omega}_{m+1}\left(\max _{\in \in[0, \tau]}\right\} f(s)\right\}\right)}{\tilde{\omega}_{m}(\tau)}\right\} \tilde{\omega}_{m}(t), \quad t \geq 0, \\
\tilde{\omega}_{j+1}(t):=\max _{\tau \in[0, t]}\left\{\frac{\left.\left.\hat{\omega}_{j+1}\left(\max _{s \in[0, \tau]}\right\} f(s)\right\}\right)}{\tilde{\omega}_{j}(\tau)}\right\} \tilde{\omega}_{j}(t), \quad t \geq 0, j=m+1, \ldots, m+n-1,
\end{array}\right.
$$

where $\hat{\omega}_{j}(t):=\max _{\tau \in[0, t]}\left\{\bar{\omega}_{j}(\tau)\right\}$ for $j=m+1, \ldots, m+n, \bar{\omega}_{i}(t):=\omega_{i}(t)+\varepsilon_{i}$ for $t \geq 0, \epsilon_{i}:=\varepsilon_{1}$ if $\omega_{i}(0)=0$ or $:=0$ if $\omega_{i}(0) \neq 0$ for $i=1,2, \ldots, m+n$, and $\varepsilon_{1}>0$ be a given very small constant.

Remark 1 If $f$ and $\omega_{i}(u)(i=1, \ldots, m)$ are continuous and nondecreasing functions on $\mathbb{R}+$ and are positive on $(0, \infty)$ such that $\omega_{1} \propto \cdots \propto \omega_{m} \propto \omega_{m+1} \circ f \propto \cdots \propto \omega_{m+n} \circ f$, then define function $\tilde{\omega}_{i}(u):=\omega_{i}(u)(i=1, \ldots, m), \tilde{\omega}_{j}(u):=\omega_{i}(f(u))(j=m+1, \ldots, m+n)$.

Theorem 2.1 Suppose that (H1)-(H6) hold, $\max _{s \in\left[b^{*}\left(t_{0}\right)-h, t_{0}\right]} \psi(s) \leq \varphi^{-1}\left((1+m+n)^{1-1 / q} \times\right.$ $\left.a\left(t_{0}\right)\right)$, and $u \in C\left(\left[b^{*}\left(t_{0}\right)-h, t_{1}\right), \mathbb{R}_{+}\right)$satisfies system (1.6) of integral inequalities. 
Then

$$
u(t) \leq \varphi^{-1}\left\{\left(W_{m+n}^{-1}\left(W_{m+n}\left(r_{m+n}(t)\right)+\int_{b_{m+n}\left(t_{0}\right)}^{b_{m+n}(t)} \tilde{g}_{m+n}(t, s) d s\right)\right)^{\frac{1}{q}}\right\}
$$

for all $t \in\left[t_{0}, T\right]$, where $W_{i}^{-1}$ is the inverse of the function

$$
W_{i}(u):=\int_{u_{i}}^{u} \frac{d x}{\tilde{\omega}_{i}^{q}\left(\varphi^{-1}\left(x^{\frac{1}{q}}\right)\right)}, \quad u \geq u_{i}>0, i=1, \ldots, m+n,
$$

$u_{i}>0$ is a given constant, $\tilde{\omega}_{i}(i=1,2, \ldots, m+n)$ are defined by $(2.1), r_{i}(t)$ is defined by $r_{1}(t):=\hat{a}(t)$ and

$$
\begin{gathered}
r_{i+1}(t):=W_{i}^{-1}\left(W_{i}\left(r_{i}(t)\right)+\int_{b_{i}\left(t_{0}\right)}^{b_{i}(t)} \tilde{g}_{i}(t, s) d s\right), \quad i=1,2, \ldots, m+n-1, \\
\tilde{g}_{i}(t, s):=(1+m+n)^{q-1} d_{i}^{q}(t)\left(\max _{\iota \in\left[t_{0}, t\right]} g_{i}(l, s)\right)^{q}, \quad t \geq t_{0}, \\
\hat{a}(t):=(1+m+n)^{q-1}\left(\max _{\tau \in\left[t_{0}, t\right]}\{a(\tau)\}\right)^{q}, d_{i}(t):=\alpha_{i}^{-\frac{1}{p}} t^{\alpha_{i} k_{i}\left(\beta_{i}-1\right)+q_{i}\left(\gamma_{i}-1\right)+1 / p} B\left(\frac{p q_{i}\left(\gamma_{i}-1\right)+1}{\alpha_{i}}, p k_{i}\left(\beta_{i}-\right.\right. \\
1)+1)^{\frac{1}{p}}, \frac{1}{p}+\frac{1}{q}=1, \text { and } T<t_{1} \text { is the largest number such that } \\
W_{i}\left(r_{i}(T)\right)+\int_{\alpha_{i}\left(t_{0}\right)}^{\alpha_{i}(T)} \max _{l \in\left[t_{0}, T\right]} f(\iota, s) d s \leq \int_{u_{i}}^{\infty} \frac{d z}{\tilde{\omega}_{i}^{q}\left(\varphi^{-1}\left(z^{\frac{1}{q}}\right)\right)}, \quad i=1,2,3, \ldots, m+n .
\end{gathered}
$$

In order to prove the theorem, we need the following lemma.

Lemma 2.1 Let $\alpha, \beta, \gamma$, and $p$ be positive constants, $a$ and $b$ be nonnegative constants. Then

$$
\int_{0}^{t}\left(t^{\alpha}-s^{\alpha}\right)^{p a(\beta-1)} s^{p b(\gamma-1)} d s=\frac{t^{\delta}}{\alpha} B\left(\frac{p b(\gamma-1)+1}{\alpha}, p a(\beta-1)+1\right), \quad t \in \mathbb{R}_{+},
$$

where $\delta:=p[a \alpha(\beta-1)+b(\gamma-1)]+1 \geq 0$.

Proof Change the variable $v: t^{\alpha} v=s^{\alpha}$, then $d s=\frac{t}{\alpha} v^{(1-\alpha) / \alpha} d v$, and we have

$$
\begin{aligned}
\int_{0}^{t}\left(t^{\alpha}-s^{\alpha}\right)^{p a(\beta-1)} s^{p b(\gamma-1)} d s & =\int_{0}^{1}\left(t^{\alpha}-t^{\alpha} v\right)^{p a(\beta-1)}\left(t v^{1 / \alpha}\right)^{p b(\gamma-1)} \frac{t}{\alpha} v^{(1-\alpha) / \alpha} d v \\
& =\frac{1}{\alpha} t^{p[a \alpha(\beta-1)+b(\gamma-1)]+1} \int_{0}^{1} v^{\frac{p b(\gamma-1)+1}{\alpha}-1}(1-v)^{p a(\beta-1)} d v \\
& =\frac{t^{\delta}}{\alpha} B\left(\frac{p b(\gamma-1)+1}{\alpha}, p a(\beta-1)+1\right), \quad t \geq 0 .
\end{aligned}
$$

This completes the proof.

Lemma 2.2 (Discrete Jensen inequality) Let If $A_{1}, \ldots, A_{n}$ be nonnegative for real numbers and $r>1$. Then

$$
\left(A_{1}+\cdots+A_{n}\right)^{r} \leq n^{r-1}\left(A_{1}^{r}+\cdots+A_{n}^{r}\right)
$$


Lemma 2.3 (see [8]) Suppose that

(C1) all $h_{i}(i=1,2, \ldots, n)$ are continuous and nondecreasing on $\mathbb{R}_{+}$and are positive on $(0, \infty)$ such that $h_{1} \propto h_{2} \propto \cdots \propto h_{m+n} ;$

(C2) $a(t)$ is continuously differentiable in $t$ and nonnegative on $\left[t_{0}, t_{1}\right)$ where $t_{0}, t_{1}$ are constants and $t_{0}<t_{1}$

(C3) all $b_{i}:\left[t_{0}, t_{1}\right) \rightarrow \mathbb{R}_{+}(i=1,2, \ldots, n)$ are continuously differentiable and nondecreasing such that $b_{i}(t) \leq t$ on $\left[t_{0}, t_{1}\right)$;

(C4) all $f_{i}(t, s), i=1, \ldots, n$, are continuous and nonnegative functions on $\left[t_{0}, t_{1}\right) \times\left[t_{0}, t_{1}\right)$. If $u(t)$ is a continuous and nonnegative function on $\left[t_{0}, t_{1}\right)$ satisfying

$$
u(t) \leq a(t)+\sum_{i=1}^{n} \int_{b_{i}\left(t_{0}\right)}^{b_{i}(t)} f_{i}(t, s) h_{i}(u(s)) d s, \quad t_{0} \leq t<t_{1}
$$

then

$$
u(t) \leq W_{n}^{-1}\left[W_{n}\left(r_{n}(t)\right)+\int_{b_{n}\left(t_{0}\right)}^{b_{n}(t)} \max _{t_{0} \leq \tau \leq t} f_{i}(\tau, s) d s\right], \quad t_{0} \leq t \leq T_{1},
$$

where for all $t \in\left[t_{0}, T_{1}\right]$, where $H_{i}^{-1}$ is the inverse of the function

$$
H_{i}(u):=\int_{u_{i}}^{u} \frac{d x}{h_{i}(x)}, \quad u \geq u_{i}>0, i=1,2, \ldots, n,
$$

$\hat{r}_{n}(t)$ is defined by $\hat{r}_{1}(t):=a\left(t_{0}\right)+\int_{t_{0}}^{t}\left|a^{\prime}(s)\right| d s$, and

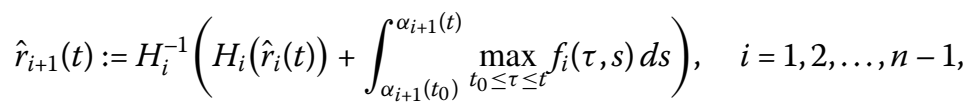

and $T_{1}<t_{1}$ is the largest number such that

$$
H_{i}\left(\hat{r}_{i}\left(T_{1}\right)\right)+\int_{\alpha_{i}\left(t_{0}\right)}^{\alpha_{i}\left(T_{1}\right)} \max _{t_{0} \leq \tau \leq t} f_{i}(\tau, s) d s \leq \int_{u_{i}}^{\infty} \frac{d z}{h_{i}(z)}, \quad i=1,2,3, \ldots, n .
$$

Proof of Theorem 2.1 First of all, we monotonize some given functions $f, \omega_{i}$, and $a$ in system (1.6) of integral inequalities. Let

$$
\tilde{f}(t):=\max _{\tau \in[0, t]}\{f(\tau)\}, \quad t \geq 0, \quad \tilde{a}(t):=\max _{\tau \in\left[t_{0}, t\right]}\{a(\tau)\}, \quad t \geq t_{0} .
$$

From (2.3) we see that the function $W_{i}$ is strictly increasing and therefore its inverse $W_{i}^{-1}$ is well defined, continuous, and increasing in its domain. The sequence $\left\{\tilde{\omega}_{i}(t)\right\}$, defined by $\omega_{i}(s)$, consists of nondecreasing nonnegative functions on $\mathbb{R}_{+}$and satisfies

$$
\begin{aligned}
& \omega_{i}(t) \leq \tilde{\omega}_{i}(t), \quad i=1,2, \ldots, m, \\
& \omega_{i}(t) \leq \hat{\omega}_{i}(t), \quad i=m+1, \ldots, m+n, \\
& \hat{\omega}_{i}(\tilde{f}(t)) \leq \tilde{\omega}_{i}(t), \quad i=m+1, \ldots, m+n .
\end{aligned}
$$


Moreover,

$$
\tilde{\omega}_{i} \propto \tilde{\omega}_{i+1}, \quad i=1,2, \ldots, m+n,
$$

because the ratios $\tilde{\omega}_{i+1}(t) / \tilde{\omega}_{i}(t), i=1,2, \ldots, m+n$, are all nondecreasing. Furthermore, let

$$
\hat{g}_{i}(t, s):=\max _{\iota \in\left[t_{0}, t\right]} g_{i}(\iota, s)
$$

which is nondecreasing in $t$ for each fixed $s$ and satisfies $\hat{g}_{i}(t, s) \geq g_{i}(t, s) \geq 0$ for all $i=$ $1,2, \ldots, m+n$. We note that $\tilde{a}(t) \geq a(t)$ and $\hat{g}_{i}(t, s) \geq f_{i}(t, s)$ and they are continuous and nondecreasing in $t$. From the monotonicity of $\tilde{f}(t)$ we obtain the inequality

$$
\begin{aligned}
\max _{\xi \in\left[c_{i}(s)-h, c_{i}(s)\right]} f(u(\xi)) & \leq \max _{\xi \in\left[c_{i}(s)-h, c_{i}(s)\right]} \tilde{f}(u(\xi)) \\
& \leq \tilde{f}\left(\max _{\xi \in\left[c_{i}(s)-h, c_{i}(s)\right]} u(\xi)\right), \quad \forall s \in\left[b^{*}\left(t_{0}\right), t_{1}\right) .
\end{aligned}
$$

From (1.6), (2.8), (2.11), and the definition of $\hat{g}_{i}(t, s)$, we obtain

$$
\begin{aligned}
\varphi(u(t)) \leq & \tilde{a}(t)+\sum_{i=1}^{m} \int_{b_{i}\left(t_{0}\right)}^{b_{i}(t)}\left(t^{\alpha_{i}}-s^{\alpha_{i}}\right)^{k_{i}\left(\beta_{i}-1\right)} s^{q_{i}\left(\gamma_{i}-1\right)} \hat{g}_{i}(t, s) \tilde{\omega}_{i}(u(s)) d s \\
& +\sum_{j=m+1}^{m+n} \int_{b_{j}\left(t_{0}\right)}^{b_{j}(t)}\left(t^{\alpha_{j}}-s^{\alpha_{j}}\right)^{k_{j}\left(\beta_{j}-1\right)} s^{q_{j}\left(\gamma_{j}-1\right)} \hat{g}_{j}(t, s) \\
& \times \hat{\omega}_{j}\left(\tilde{f}\left(\max _{\xi \in\left[c_{j}(s)-h, c_{j}(s)\right]} u(\xi)\right)\right) d s \\
\leq & \tilde{a}(t)+\sum_{i=1}^{m} \int_{b_{i}\left(t_{0}\right)}^{b_{i}(t)}\left(t^{\alpha_{i}}-s^{\alpha_{i}}\right)^{k_{i}\left(\beta_{i}-1\right)} s^{q_{i}\left(\gamma_{i}-1\right)} \hat{g}_{i}(t, s) \tilde{\omega}_{i}(u(s)) d s \\
& +\sum_{j=m+1}^{m+n} \int_{b_{j}\left(t_{0}\right)}^{b_{j}(t)}\left(t^{\alpha_{j}}-s^{\alpha_{j}}\right)^{k_{j}\left(\beta_{j}-1\right)} s^{q_{j}\left(\gamma_{j}-1\right)} \hat{g}_{j}(t, s) \\
& \times \tilde{\omega}_{j}\left(\max _{\xi \in\left[c_{j}(s)-h, c_{j}(s)\right]} u(\xi)\right) d s, \quad t \in\left[b_{j}\left(t_{0}\right), t_{1}\right), \\
u(t) \leq \psi( & t), \quad t \in\left[b^{*}\left(t_{0}\right)-h, t_{0}\right] .
\end{aligned}
$$

Let $\frac{1}{p}+\frac{1}{q}=1, p>1$, then $q>0$. Since $p q_{i}\left(\gamma_{i}-1\right)+1>0, p k_{i}\left(\beta_{i}-1\right)+1>0$, and $\frac{1}{p}+k_{i} \alpha_{i}\left(\beta_{i}-\right.$ $1)+q_{i}\left(\gamma_{i}-1\right) \geq 0$ for $i=1, \ldots, m+n$. By Lemma 2.1, Hölder's inequality, and (2.12) we get for $t \in\left[t_{0}, t_{1}\right)$

$$
\begin{aligned}
\varphi(u(t)) \leq & \tilde{a}(t)+\sum_{i=1}^{m}\left(\int_{b_{i}\left(t_{0}\right)}^{b_{i}(t)}\left(t^{\alpha_{i}}-s^{\alpha_{i}}\right)^{p k_{i}\left(\beta_{i}-1\right)} s^{p q_{i}\left(\gamma_{i}-1\right)} d s\right)^{\frac{1}{p}}\left(\int_{b_{i}\left(t_{0}\right)}^{b_{i}(t)} \hat{g}_{i}^{q}(t, s) \tilde{\omega}_{i}^{q}(u(s)) d s\right)^{\frac{1}{q}} \\
& +\sum_{j=m+1}^{m+n}\left(\int_{b_{j}\left(t_{0}\right)}^{b_{j}(t)}\left(t^{\alpha_{j}}-s^{\alpha_{j}}\right)^{p k_{j}\left(\beta_{j}-1\right)} s^{p q_{j}\left(\gamma_{j}-1\right)} d s\right)^{\frac{1}{p}} \\
& \times\left(\int_{b_{j}\left(t_{0}\right)}^{b_{j}(t)} \hat{g}_{j}^{q}(t, s) \tilde{\omega}_{j}^{q}\left(\max _{\xi \in\left[c_{j}(s)-h, c_{j}(s)\right]} u(\xi)\right) d s\right)^{\frac{1}{q}}
\end{aligned}
$$




$$
\begin{aligned}
& \leq \tilde{a}(t)+\sum_{i=1}^{m}\left(\int_{0}^{t}\left(t^{\alpha_{i}}-s^{\alpha_{i}}\right)^{p k_{i}\left(\beta_{i}-1\right)} s^{p q_{i}\left(\left(\gamma_{i}-1\right)\right)} d s\right)^{\frac{1}{p}}\left(\int_{b_{i}\left(t_{0}\right)}^{b_{i}(t)} \hat{g}_{i}^{q}(t, s) \tilde{\omega}_{i}^{q}(u(s)) d s\right)^{\frac{1}{q}} \\
& \quad+\sum_{j=m+1}^{m+n}\left(\int_{0}^{t}\left(t^{\alpha_{j}}-s^{\alpha_{j}}\right)^{p k_{j}\left(\beta_{j}-1\right)} s^{p q_{j}\left(\gamma_{j}-1\right)} d s\right)^{\frac{1}{p}} \\
& \quad \times\left(\int_{b_{j}\left(t_{0}\right)}^{b_{j}(t)} \hat{g}_{j}^{q}(t, s) \tilde{\omega}_{j}^{q}\left(\max _{\xi \in\left[c_{j}(s)-h_{,}(s)\right]} u(\xi)\right) d s\right)^{\frac{1}{q}} \\
& \leq \tilde{a}(t)+\sum_{i=1}^{m} d_{i}(t)\left(\int_{b_{i}\left(t_{0}\right)}^{b_{i}(t)} \hat{g}_{i}^{q}(t, s) \tilde{\omega}_{i}^{q}(u(s)) d s\right)^{\frac{1}{q}} \\
& \quad+\sum_{j=m+1}^{m+n} d_{j}(t)\left(\int_{b_{j}\left(t_{0}\right)}^{b_{j}(t)} \hat{g}_{j}^{q}(t, s) \tilde{\omega}_{j}^{q}\left(\max _{\xi \in\left[c_{j}(s)-h, c_{j}(s)\right]} u(\xi)\right) d s\right)^{\frac{1}{q}},
\end{aligned}
$$

where we use $0 \leq b_{i}(t) \leq t$ and the definition of $d_{i}(t)$.

By Lemma 2.2 and (2.13), we get for $t \in\left[t_{0}, t_{1}\right)$

$$
\begin{aligned}
\varphi^{q}(u(t)) \leq & (1+m+n)^{q-1}\left[\tilde{a}^{q}(t)+\sum_{i=1}^{m} d_{i}^{q}(t) \int_{b_{i}\left(t_{0}\right)}^{b_{i}(t)} \hat{g}_{i}^{q}(t, s) \tilde{\omega}_{i}^{q}(u(s)) d s\right. \\
& \left.+\sum_{j=m+1}^{m+n} d_{j}^{q}(t) \int_{b_{j}\left(t_{0}\right)}^{b_{j}(t)} \hat{g}_{j}^{q}(t, s) \tilde{\omega}_{j}^{q}\left(\max _{\xi \in\left[c_{j}(s)-h, c_{j}(s)\right]} u(\xi)\right) d s\right] .
\end{aligned}
$$

Then from (2.4), we see that $\hat{r}_{1}(t)$ is nondecreasing on $\left[t_{0}, t_{1}\right)$. By the definition of $\tilde{g}_{i}(t, s)$ and $\hat{r}_{1}(t)$, and (2.14), we have

$$
\begin{aligned}
& \varphi^{q}(u(t)) \leq \hat{r}_{1}(t)+\sum_{i=1}^{m} \int_{b_{i}\left(t_{0}\right)}^{b_{i}(t)} \tilde{g}_{i}(t, s) \tilde{\omega}_{i}^{q}(u(s)) d s \\
& \quad+\sum_{j=m+1}^{m+n} \int_{b_{j}\left(t_{0}\right)}^{b_{j}(t)} \tilde{g}_{j}(t, s) \tilde{\omega}_{j}^{q}\left(\max _{\xi \in\left[c_{j}(s)-h, c_{j}(s)\right]} u(\xi)\right) d s, \quad t \in\left[t_{0}, t_{1}\right), \\
& u(t) \leq \psi(t), \quad t \in\left[b^{*}\left(t_{0}\right)-h, t_{0}\right] .
\end{aligned}
$$

Consider the auxiliary system of inequalities with (2.15)

$$
\begin{aligned}
\varphi^{q}(u(t)) \leq & \hat{r}_{1}(\sigma)+\sum_{i=1}^{m} \int_{b_{i}\left(t_{0}\right)}^{b_{i}(t)} \tilde{g}_{i}(\sigma, s) \tilde{\omega}_{i}^{q}(u(s)) d s \\
& +\sum_{j=m+1}^{m+n} \int_{b_{j}\left(t_{0}\right)}^{b_{j}(t)} \tilde{g}_{j}(\sigma, s) \tilde{\omega}_{j}^{q}\left(\max _{\xi \in\left[c_{j}(s)-h, c_{j}(s)\right]} u(\xi)\right) d s,
\end{aligned}
$$

for all $t \in\left[t_{0}, \sigma\right]$, where $\sigma$ is chosen arbitrarily such that $t_{0} \leq \sigma \leq T_{1}$.

Notice that $\max _{s \in\left[b^{*}\left(t_{0}\right)-h, t_{0}\right]} \psi(s) \leq \varphi^{-1}\left(\hat{r}_{1}^{1 / q}(\sigma)\right)$ because $\max _{\left.s \in U\left(t_{0}\right)-h, t_{0}\right]} \psi(s) \leq \varphi^{-1}((1+$ $\left.m+n)^{\frac{p-1}{p}} a^{\frac{1}{q}}\left(t_{0}\right)\right) \leq \varphi^{-1}\left(\hat{r}_{1}(\sigma)\right)$. Define a function $z(t):\left[B^{*}\left(t_{0}\right)-h, \sigma\right] \rightarrow \mathbb{R}_{+}$such that

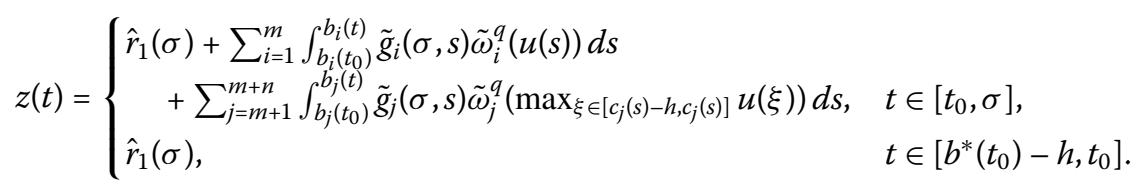


Clearly, $z(t)$ is nondecreasing. By (2.16) and the definition of $z(t)$ we have

$$
u(t) \leq \varphi^{-1}\left(z^{\frac{1}{q}}(t)\right), \quad t \in\left[b^{*}\left(t_{0}\right)-h, \sigma\right] .
$$

Since $z(t)$ is nondecreasing, from (2.17) we obtain

$$
\begin{aligned}
\max _{\xi \in\left[c_{j}(s)-h, c_{j}(s)\right]} u(\xi) & \leq \max _{\xi \in\left[c_{j}(s)-h, c_{j}(s)\right]} \varphi^{-1}\left(z^{\frac{1}{q}}(\xi)\right) \\
& \leq \varphi^{-1}\left(z^{\frac{1}{q}}\left(c_{j}(s)\right)\right) \leq \varphi^{-1}\left(z^{\frac{1}{q}}(s)\right), \quad s \in\left[b_{j}\left(t_{0}\right), b_{j}(\sigma)\right] .
\end{aligned}
$$

It follows from (2.17), (2.18), and the definition of $z(t)$ that

$$
\begin{aligned}
z(t) \leq & \hat{r}_{1}(\sigma)+\sum_{i=1}^{m} \int_{b_{i}\left(t_{0}\right)}^{b_{i}(t)} \tilde{g}_{i}(\sigma, s) \tilde{\omega}_{i}^{q}\left(\varphi^{-1}\left(z^{\frac{1}{q}}(s)\right)\right) d s \\
& +\sum_{j=m+1}^{m+n} \int_{b_{j}\left(t_{0}\right)}^{b_{j}(t)} \tilde{g}_{j}(\sigma, s) \tilde{\omega}_{j}^{q}\left(\varphi^{-1}\left(z^{\frac{1}{q}}(s)\right)\right) d s, \quad t \in\left[t_{0}, \sigma\right] .
\end{aligned}
$$

In order to demonstrate the basic condition of monotonicity, let $e(t):=\varphi^{-1}\left(t^{\frac{1}{q}}\right)$, which is clearly a continuous and nondecreasing function on $\mathbb{R}_{+}$. Thus, for each $i, \tilde{\omega}_{i}(e(t))$ is continuous and nondecreasing on $\mathbb{R}_{+}$and $\tilde{\omega}_{i}(e(t))>0$ for $t>0$. Moreover, since $\tilde{\omega}_{i}(t) \propto \tilde{\omega}_{i+1}(t)$, we see that the ratio $\tilde{\omega}_{i+1}(b(t)) / \tilde{\omega}_{i}(e(t))$ is also a continuous and nondecreasing function on $\mathbb{R}_{+}$and satisfies $\tilde{\omega}_{i}(e(t))>0$ for $t>0$, implying that $\tilde{\omega}_{i}^{q}(e(t)) \propto \tilde{\omega}_{i+1}^{q}(e(t)), i=2, \ldots, m+n-1$. Applying Lemma 2.3 to the case that $f_{i}(t, s)=\tilde{g}_{i}(\sigma, s), a(t)=\hat{r}_{1}(\sigma)$, and $\omega_{i}(t)=\tilde{\omega}_{i}^{q}\left(\varphi^{-1}\left(t^{\frac{1}{q}}\right)\right)$, $i=1,2, \ldots, m+n$, from (2.19) we obtain

$$
z(t) \leq W_{m+n}^{-1}\left(W_{m+n}\left(\hat{r}_{m+n}(\sigma, t)\right)+\int_{b_{m+n}\left(t_{0}\right)}^{b_{m+n}(t)} \tilde{g}_{m+n}(\sigma, s) d s\right)
$$

for all $t_{0} \leq t \leq \min \left\{\sigma, T_{1}\right\}$, where

$$
\begin{aligned}
& \tilde{r}_{1}(\sigma, t):=\hat{r}_{1}(\sigma) \\
& \tilde{r}_{i+1}(\sigma, t):=W_{i}^{-1}\left(W_{i}\left(\tilde{r}_{i}(\sigma, t)\right)+\int_{b_{i}\left(t_{0}\right)}^{b_{i}(t)} \tilde{g}_{i}(\sigma, s) d s\right), \quad i=1,2, \ldots, m+n-1,
\end{aligned}
$$

and $T_{1}<t_{1}$ is the largest number such that

$$
W_{i}\left(\tilde{r}_{i}\left(\sigma, T_{1}\right)\right)+\int_{b_{i}\left(t_{0}\right)}^{b_{i}\left(T_{1}\right)} \tilde{g}_{i}(\sigma, s) d s \leq \int_{u_{i}}^{\infty} \frac{d z}{\tilde{\omega}_{i}^{q}\left(\varphi^{-1}\left(z^{\frac{1}{q}}\right)\right)}
$$

for $i=1,2,3, \ldots, m+n$. Notice that $T \leq T_{1}$. In fact, $W_{i}$ is strictly increasing by (2.3), so its inverse $W_{i}^{-1}$ is continuous and increasing in its corresponding domain by (2.3). It follows from (2.21) and the definition of $\tilde{g}_{i}(\sigma, s)$ that $\tilde{r}_{i}(\sigma, t)$ and $\tilde{g}_{i}(\sigma, s)$ are nondecreasing in $\sigma$. Thus, $T_{1}$ satisfying (2.22) gets smaller as $\sigma$ is chosen larger. In particular, $T_{1}$ satisfies the same equation (2.6) as $T$ when $\sigma=T$. It follows from (2.17) and (2.20) that

$$
u(t) \leq \varphi^{-1}\left(\left(W_{m+n}^{-1}\left(W_{m+n}\left(\tilde{r}_{m+n}(\sigma, t)\right)+\int_{b_{m+n}\left(t_{0}\right)}^{b_{m+n}(t)} \tilde{g}_{m+n}(\sigma, s) d s\right)\right)^{1 / q}\right)
$$


Taking $t=\sigma$ in (2.23), we have

$$
u(\sigma) \leq \varphi^{-1}\left(\left(W_{m+n}^{-1}\left(W_{m+n}\left(\tilde{r}_{m+n}(\sigma, \sigma)\right)+\int_{b_{m+n}\left(t_{0}\right)}^{b_{m+n}(\sigma)} \tilde{g}_{m+n}(\sigma, s) d s\right)\right)^{1 / q}\right)
$$

for $0 \leq \sigma \leq T$. It is easy to verify $\tilde{r}_{i}(\sigma, \sigma)=\hat{r}_{i}(\sigma)$.

Thus, (2.24) can be written

$$
u(\sigma) \leq \varphi^{-1}\left(\left(W_{m+n}^{-1}\left(W_{m+n}\left(\tilde{r}_{m+n}(\sigma)\right)+\int_{b_{m+n}\left(t_{0}\right)}^{b_{m+n}(\sigma)} \tilde{g}_{m+n}(\sigma, s) d s\right)\right)^{1 / q}\right)
$$

for $0 \leq \sigma \leq T$. Since $\sigma$ is arbitrary, replacing $\sigma$ with $t$, we get

$$
u(t) \leq \varphi^{-1}\left(\left(W_{m+n}^{-1}\left(W_{m+n}\left(\tilde{r}_{m+n}(t)\right)+\int_{b_{m+n}\left(t_{0}\right)}^{b_{m+n}(t)} \tilde{g}_{m+n}(t, s) d s\right)\right)^{1 / q}\right)
$$

for $t_{0} \leq t \leq T$. This completes the proof.

Corollary 2.1 Suppose that (H1)-(H6) hold, and $u \in C\left(\left(b^{*}\left(t_{0}\right)-h, t_{1}\right), \mathbb{R}_{+}\right)$satisfies

$$
\begin{aligned}
& \varphi(u(t)) \leq c+\sum_{i=1}^{m} \int_{b_{i}\left(t_{0}\right)}^{b_{i}(t)}\left(t^{\alpha_{i}}-s^{\alpha_{i}}\right)^{k_{i}\left(\beta_{i}-1\right)} s^{q_{i}\left(\gamma_{i}-1\right)} g_{i}(t, s) \omega_{i}(u(s)) d s \\
&+\sum_{j=m+1}^{m+n} \int_{b_{j}\left(t_{0}\right)}^{b_{j}(t)}\left(t^{\alpha_{j}}-s^{\alpha_{j}}\right)^{k_{j}\left(\beta_{j}-1\right)} s^{q_{j}\left(\gamma_{j}-1\right)} g_{j}(t, s) \\
& \times \omega_{j}\left(\max _{\xi \in\left[c_{j}(s)-h, c_{j}(s)\right]} f(u(\xi))\right) d s, \quad t \in\left[t_{0}, t_{1}\right), \\
& u(t) \leq \psi(t), \quad t \in\left[b^{*}\left(t_{0}\right)-h, t_{0}\right],
\end{aligned}
$$

where $c \geq 0$ is a constant. Then

$$
u(t) \leq \varphi^{-1}\left\{\left(W_{m+n}^{-1}\left(W_{m+n}\left(\bar{r}_{m+n}(t)\right)+\int_{b_{m+n}\left(t_{0}\right)}^{b_{m+n}(t)} \tilde{g}_{m+n}(t, s) d s\right)\right)^{1 / q}\right\}
$$

for all $t \in\left[t_{0}, t_{3}\right)$, where $W_{i}^{-1}$ is the inverse of $W_{i}, W_{i}$ is defined in (2.3), $\bar{r}_{i}(t)$ is defined by $\bar{r}_{1}(t):=\varphi^{q}(M)$ and

$$
\bar{r}_{i+1}(t):=W_{i}^{-1}\left(W_{i}\left(\bar{r}_{i}(t)\right)+\int_{b_{i}\left(t_{0}\right)}^{b_{i}(t)} \tilde{g}_{i}(t, s) d s\right), \quad i=1,2, \ldots, m+n-1,
$$

$M:=\max \left(\max _{s \in\left[b^{*}\left(t_{0}\right)-h, t_{0}\right]} \psi(s), \varphi^{-1}\left((1+m+n)^{1-1 / q} c\right)\right), \frac{1}{p}+\frac{1}{q}=1, t_{3}<t_{1}$ is the largest number such that

$$
W_{i}\left(\bar{r}_{i}\left(t_{3}\right)\right)+\int_{b_{i}\left(t_{0}\right)}^{b_{i}\left(t_{3}\right)} \tilde{g}_{i}\left(t_{3}, s\right) d s \leq \int_{u_{i}}^{\infty} \frac{d z}{\tilde{\omega}_{i}^{q}\left(\varphi^{-1}\left(z^{\frac{1}{q}}\right)\right)}, \quad i=1,2,3, \ldots, m+n
$$

and $\tilde{\omega}_{i}$ and $\tilde{g}_{i}$ are defined by (2.1) and (2.5), respectively. 
Proof From (2.27) and the definition of $M$ we get

$$
\begin{aligned}
& \varphi(u(t)) \leq(1+m+n)^{1 / q-1} \varphi(M) \\
&+\sum_{i=1}^{m} \int_{b_{i}\left(t_{0}\right)}^{b_{i}(t)}\left(t^{\alpha_{i}}-s^{\alpha_{i}}\right)^{k_{i}\left(\beta_{i}-1\right)} s^{q_{i}\left(\gamma_{i}-1\right)} g_{i}(t, s) \omega_{i}(u(s)) d s \\
&+\sum_{j=m+1}^{m+n} \int_{b_{j}\left(t_{0}\right)}^{b_{j}(t)}\left(t^{\alpha_{j}}-s^{\alpha_{j}}\right)^{k_{j}\left(\beta_{j}-1\right)} s^{q_{j}\left(\gamma_{j}-1\right)} g_{j}(t, s) \\
& \times \omega_{j}\left(\max _{\xi \in\left[c_{j}(s)-h, c_{j}(s)\right]} f(u(\xi))\right) d s, \quad t \in\left[t_{0}, t_{1}\right), \\
& u(t) \leq M, \quad t \in\left[b^{*}\left(t_{0}\right)-h, t_{0}\right] .
\end{aligned}
$$

Then from (2.31) we obtain $(2.28)$ by Theorem 2.1 , where we choose $a(t)(1+m+$ $n)^{1 / q-1} \varphi(M)$. This completes the proof.

\section{Applications}

In this section, we apply our result to estimate solutions for the nonlinear integral equation and integral equation with a weakly singular kernel and maxima separately.

\subsection{Differential equation with the maxima}

Consider a system of differential equations with maxima

$$
\begin{cases}x^{\prime}(t)=F\left(t, x(t), \max _{s \in[\beta(t), \alpha(t)]} x(s)\right)+c \int_{0}^{t}(t-s)^{-\lambda} x^{\prime}(s) d s, & t \geq t_{0}, \\ x(t)=\psi_{1}(t), & t \in\left[\alpha\left(t_{0}\right)-h, t_{0}\right],\end{cases}
$$

where $c, \lambda(0<\lambda<1), t_{0} \geq 0$, and $h>0$ are constants, $\psi_{1} \in C\left(\left[\alpha\left(t_{0}\right)-h, t_{0}\right], \mathbb{R}\right), F \in C\left(\mathbb{R}_{+} \times\right.$ $\left.\mathbb{R}^{2}, \mathbb{R}\right), \alpha, \beta \in C^{1}\left(\left[t_{0}, \infty\right), \mathbb{R}_{+}\right), \alpha(t)$ is a nondecreasing function, $\beta(t) \leq t, \alpha(t) \leq t$, and $0<$ $\alpha(t)-\beta(t) \leq h$ for $t \geq t_{0}$.

Equation (3.1) is more general than the equation considered in Section 3 of [26] so that the results of the integral inequalities obtained in [26] do not work. We will give an estimate for solutions of system (3.1).

Corollary 3.1 Suppose in system (3.1) that

$$
|F(t, x, y)| \leq h_{1}(t)|x| \mu_{1}(|x|)+h_{2}(t)|y| \mu_{2}(|y|)+h_{3}(t), \quad t \geq 0, x, y \in \mathbb{R},
$$

where $h_{i} \in C\left(\mathbb{R}_{+}, \mathbb{R}_{+}\right)(i=1,2,3)$, all $\mu_{i}(i=1,2)$ are continuous and nondecreasing on $\mathbb{R}_{+}$ and are positive on $(0, \infty)$ such that $\mu_{1} \propto \mu_{2}, 1<p<1 / \lambda, \frac{1}{p}+\frac{1}{q}=1$. For given $u_{1}>0$ and $u_{2}>0$, let

$$
\begin{array}{ll}
Q_{1}(u):=\int_{u_{1}}^{u_{2}} \frac{d s}{s^{q} \mu_{1}^{q}\left(s^{1 / q}\right)} d s, & u \geq u_{1}>0, \\
Q_{2}(u):=\int_{u_{2}}^{u} \frac{d s}{s^{q} \mu_{2}^{q}\left(s^{1 / q}\right)} d s, & u \geq u_{2}>0 .
\end{array}
$$


Then every solution $x\left(t, t_{0}, \psi_{1}\right)$ of system (3.1) has the estimate

$$
\left|x\left(t, t_{0}, \psi_{1}\right)\right| \leq\left(Q_{2}^{-1}\left(Q_{2}\left(\gamma_{2}(t)\right)+4^{q-1} t^{q / p} \int_{t_{0}}^{t} h_{2}^{q}(s) d s\right)\right)^{1 / q}, \quad \forall t \in\left[t_{0}, t^{*}\right]
$$

where $\gamma_{i}(t)$ are defined by $\gamma(t):=4^{q-1} N^{q}(t) \exp \left(4^{q-1} t^{q(1-p \lambda) / p+1}|c|^{q} B(1,1-p \lambda)^{q / p}\right)$ and

$$
\begin{aligned}
& \gamma_{1}(t):=Q_{1}^{-1}\left(Q_{1}(\gamma(t))+4^{q-1} t^{q / p} \int_{t_{0}}^{t} h_{1}^{q}(s) d s\right), \\
& \gamma_{2}(t):=Q_{1}^{-1}\left(Q_{2}\left(\gamma_{1}(t)\right)+4^{q-1} t^{q / p} \int_{t_{0}}^{t} h_{2}^{q}(s) d s\right),
\end{aligned}
$$

$N(t):=\max _{s \in\left[\alpha\left(t_{0}\right)-h, t_{0}\right]}\left|\psi_{1}(s)\right|+\left|\psi_{1}\left(t_{0}\right)\right|\left(1+|c| t^{1-\lambda} /(1-\lambda)\right)+\int_{t_{0}}^{t}\left|h_{3}(s)\right| d s$ for $t \geq t_{0}$, and $t^{*}$ is the largest number such that

$$
\begin{aligned}
& Q_{1}\left(\gamma_{1}\left(t^{*}\right)\right)+4^{q-1} t^{q / p} \int_{t_{0}}^{t^{*}} h_{1}^{q}(s) d s \leq \int_{u_{1}}^{\infty} \frac{d s}{s^{q} \mu_{1}^{q}\left(s^{1 / q}\right)}, \\
& Q_{2}\left(r_{2}\left(t^{*}\right)\right)+4^{q-1} t^{q / p} \int_{t_{0}}^{t^{*}} h_{2}^{q}(s) d s \leq \int_{u_{2}}^{\infty} \frac{d s}{s^{q} \mu_{2}^{q}\left(s^{1 / q}\right)} .
\end{aligned}
$$

Proof Let $M=\max _{s \in\left[\alpha\left(t_{0}\right)-h, t_{0}\right]}\left|\psi_{1}(s)\right|$ and $x(t)=x\left(t, t_{0}, \psi_{1}\right)$, the solution of system (3.1) defined for all $t \geq \alpha\left(t_{0}\right)-h$. The function $x(t)$ satisfies the following integral equation:

$$
\begin{aligned}
x(t)= & \psi_{1}\left(t_{0}\right)\left(1-c\left(t-t_{0}\right)^{1-\lambda} /(1-\lambda)\right)+\int_{t_{0}}^{t} F\left(s, x(s), \max _{\xi \in[\beta(s), \alpha(s)]} x(\xi)\right) d s \\
& +c \int_{t_{0}}^{t}(t-s)^{-\lambda} x(s) d s, \quad t \geq t_{0}, \\
x(t)= & \psi_{1}(t), \quad t \in\left[\alpha\left(t_{0}\right)-h, t_{0}\right] .
\end{aligned}
$$

By (3.2) and the definition of $N(t)$, we get from (3.5)

$$
\begin{aligned}
|x(t)| \leq & \left|\psi_{1}\left(t_{0}\right)\right|\left(1+|c| t^{1-\lambda} /(1-\lambda)\right)+\int_{t_{0}}^{t}\left|F\left(t, x(s), \max _{\xi \in[\beta(s), \alpha(s)]} x(\xi)\right)\right| d s \\
\leq & \left|\psi_{1}\left(t_{0}\right)\right|\left(1+|c| t^{1-\lambda} /(1-\lambda)\right)+\int_{t_{0}}^{t} h_{3}(s) d s+M+|c| \int_{t_{0}}^{t}(t-s)^{-\lambda}|x(s)| d s \\
& +\int_{t_{0}}^{t} h_{1}(s) \mu_{1}(|x(s)|)|x(s)| d s \\
& +\int_{t_{0}}^{t} h_{2}(s) \mu_{2}\left(\max _{\xi \in[\beta(s), \alpha(s)]}|x(\xi)|\right)\left(\max _{\xi \in[\beta(s), \alpha(s)]}|x(\xi)|\right) d s \\
\leq & N(t)+|c| \int_{t_{0}}^{t}(t-s)^{-\lambda}|x(s)| d s+\int_{t_{0}}^{t} h_{1}(s) \mu_{1}(|x(s)|)|x(s)| d s \\
& +\int_{t_{0}}^{t} h_{2}(s) \mu_{2}\left(\max _{\xi \in[\beta(s), \alpha(s)]}|x(\xi)|\right)\left(\max _{\xi \in[\beta(s), \alpha(s)]}|x(\xi)|\right) d s, \quad t \geq t_{0}, \\
|x(t)| \leq & \left|\psi_{1}(t)\right| \leq M, \quad t \in\left[\alpha\left(t_{0}\right)-h, t_{0}\right] .
\end{aligned}
$$


Set $u(t):=|x(t)|$ for $t \in\left[\alpha\left(t_{0}\right)-h, \infty\right)$. Then, using the inequality $\max _{\xi \in[\beta(s), \alpha(s)]} u(\xi) \leq$ $\max _{\xi \in[\alpha(s)-h, \alpha(s)]} u(\xi)$, we obtain

$$
\begin{aligned}
u(t) \leq & N(t)+\int_{t_{0}}^{t}(t-s)^{-\lambda} u(s) d s+\int_{t_{0}}^{t} h_{1}(s) \mu_{1}(u(s)) u(s) d s \\
& +\int_{t_{0}}^{t} h_{2}(s) \mu_{2}\left(\max _{\xi \in[\alpha(s)-h, \alpha(s)]} u(\xi)\right)\left(\max _{\xi \in[\alpha(s)-h, \alpha(s)]} u(\xi)\right) d s, \quad t \geq t_{0}, \\
u(t) \leq & M, \quad t \in\left[\alpha\left(t_{0}\right)-h, t_{0}\right] .
\end{aligned}
$$

Using our Theorem 2.1 to the specified $m=2, n=1, \varphi(u)=u, a(t)=N(t), \alpha_{i}=1(i=1,2,3)$, $g_{1}(t, s)=1, g_{i}(t, s)=h_{i-1}(s)(i=2,3), b_{i}(t)=t(i=1,2,3), \alpha_{i}=1(i=1,2,3), k_{1}=1, q_{1}=0$, $q_{i}=k_{i}=0(i=2,3), \beta_{1}=1-\lambda, \omega_{1}(s)=s, \omega_{2}(s)=s \mu_{1}(s), \omega_{3}(s)=s \mu_{2}(s)$, since $\mu_{1} \propto \mu_{2}$, we see that the ratio $\omega_{1} \propto \omega_{2} \propto \omega_{3}$, and from (3.7) we obtain

$$
u(t) \leq\left(Q_{2}^{-1}\left(Q_{2}\left(\gamma_{2}(t)\right)+4^{q-1} t^{\frac{q}{p}} \int_{t_{0}}^{t} h_{2}(s) d s\right)\right)^{1 / q}
$$

for all $t \in\left[t_{0}, t^{*}\right]$, where $t^{*}$ is given in (3.4). Inequality (3.8) proves the validity of inequality (3.3).

Next, we discuss the uniqueness of solutions for system (3.1).

\section{Corollary 3.2 Suppose that}

$$
\left|F\left(t, x_{1}, y_{1}\right)-F\left(t, x_{2}, y_{2}\right)\right| \leq h_{1}(t)\left|x_{1}-x_{2}\right|+h_{2}(t)\left|y_{1}-y_{2}\right|
$$

for all $t \geq t_{0}$ and all $x_{i}, y_{i} \in \mathbb{R}(i=1,2)$, where $h_{i} \in C\left(\left[t_{0}, \infty\right), \mathbb{R}_{+}\right)$. Then system (3.1) has at most one solution on $\left[t_{0}, t_{1}\right)$.

Proof Assume that (3.1) has two different solutions $u(t)=u\left(t, t_{0}, \psi_{1}\right)$ and $v(t)=v\left(t, t_{0}, \psi_{1}\right)$, defined for $t \geq \alpha\left(t_{0}\right)-h$. Then $u(t)$ and $v(t)$ satisfy the integral equations defined for all $t \geq \alpha\left(t_{0}\right)-h$. The two functions $u(t)$ and $v(t)$ satisfy the integral equations

$$
\begin{aligned}
u(t)= & \psi_{1}\left(t_{0}\right)\left(1-c t^{1-\lambda} /(1-\lambda)\right)+\int_{t_{0}}^{t} F\left(t, u(s), \max _{\xi \in[\beta(s), \alpha(s)]} u(\xi)\right) d s \\
& +\int_{t_{0}}^{t}(t-s)^{-\lambda} u(s) d s, \quad t \geq t_{0}, \\
v(t)= & \psi_{1}\left(t_{0}\right)\left(1-c t^{1-\lambda} /(1-\lambda)\right)+\int_{t_{0}}^{t} F\left(t, v(s), \max _{\xi \in[\beta(s), \alpha(s)]} v(\xi)\right) d s \\
& +\int_{t_{0}}^{t}(t-s)^{-\lambda} v(s) d s, \quad t \geq t_{0},
\end{aligned}
$$

and $u(t)=v(t)=\psi_{1}(t)$ for $t \in\left[\alpha\left(t_{0}\right)-h, t_{0}\right]$. It implies that

$$
\begin{aligned}
|u(t)-v(t)| \leq & \int_{t_{0}}^{t}\left|F\left(t, u(s), \max _{\xi \in[\beta(s), \alpha(s)]} u(\xi)\right)-F\left(t, v(s), \max _{\xi \in[\beta(s), \alpha(s)]} v(\xi)\right)\right| D s \\
& +\int_{t_{0}}^{t}(t-s)^{-\lambda}|u(s)-v(s)| d s
\end{aligned}
$$




$$
\begin{aligned}
\leq & \int_{t_{0}}^{t}(t-s)^{-\lambda}|u(s)-v(s)| d s+\int_{t_{0}}^{t} h_{1}(s)(|u(s)-v(s)|) d s \\
& +\int_{t_{0}}^{t} h_{2}(s)\left|\max _{\xi \in[\beta(s), \alpha(s)]} u(\xi)-\max _{\xi \in[\beta(s), \alpha(s)]} v(\xi)\right| d s \\
\leq & \int_{t_{0}}^{t}(t-s)^{-\lambda}|u(s)-v(s)| d s+\int_{t_{0}}^{t} h_{1}(s)|u(s)-v(s)| d s \\
& +\int_{t_{0}}^{t} h_{2}(s) \max _{\xi \in[\beta(s), \alpha(s)]}|u(\xi)-v(\xi)| d s, \quad \forall t \geq t_{0} .
\end{aligned}
$$

Let $\phi(t):=|u(t)-v(t)|$ for $t \geq \alpha\left(t_{0}\right)-h$. Noting that

$$
\max _{\xi \in[\beta(s), \alpha(s)]} u(\xi) \leq \max _{\xi \in[\alpha(s)-h, \alpha(s)]} u(\xi),
$$

from (3.11) we obtain

$$
\begin{aligned}
\phi(t) \leq & \varepsilon+\int_{t_{0}}^{t}(t-s)^{-\lambda} \phi(s) d s+\int_{t_{0}}^{t} h_{1}(s) \phi(s) d s \\
& +\int_{t_{0}}^{t} h_{2}(s) \max _{\xi \in[\alpha(s)-h, \alpha(s)]} \phi(\xi) d s, \quad t \geq t_{0}, \\
\phi(t) \leq & 0, \quad t \in\left[\alpha\left(t_{0}\right)-h, t_{0}\right] .
\end{aligned}
$$

Here, $\varepsilon$ is an arbitrary positive number, which is the formula of the system of integral inequalities (2.27). Applying our Corollary 2.1 to (3.12), we have

$$
\begin{aligned}
\phi(t) \leq & 4^{1-1 / q} \varepsilon \exp \left(\frac { 1 } { q } \left(4 ^ { q - 1 } \left(B^{q / p}(1,1-p \lambda) t^{q(1-p \lambda) / p+1}+t^{q / p} \int_{t_{0}}^{t} h_{1}^{q}(s) d s\right.\right.\right. \\
& \left.\left.\left.+t^{q / p} \int_{t_{0}}^{t} h_{2}^{q}(s) d s\right)\right)\right),
\end{aligned}
$$

$1<p<1 / \lambda, \frac{1}{p}+\frac{1}{q}=1$, letting $\varepsilon \rightarrow 0$, we obtain $|u(t)-v(t)| \leq 0$, which implies that $u(t)=v(t)$ for all $t \in\left[t_{0}, t_{1}\right)$. The uniqueness is proved.

\subsection{Integral equation with maxima}

Consider the system of integral equations with maxima

$$
\left\{\begin{aligned}
x(t)= & a(t)+\int_{t_{0}}^{t}(t-s)^{\beta_{1}-1} s^{\gamma_{1}-1} f_{1}(t, s, x(s)) d s \\
& \left.+\int_{t_{0}}^{t}(t-s)^{\beta_{2}-1} s^{\gamma_{2}-1} f_{2}\left(t, s, \max _{\xi \in[\beta(s), \alpha(s)]} x(\xi)\right)\right) d s, \quad t \geq t_{0}, \\
x(t)= & \psi(t), \quad t \in\left[\alpha\left(t_{0}\right)-h, t_{0}\right],
\end{aligned}\right.
$$

where $\psi \in C\left(\left[\alpha\left(t_{0}\right)-h, t_{0}\right], \mathbb{R}\right), f \in C\left(\mathbb{R}_{+}^{2} \times \mathbb{R}, \mathbb{R}\right), t_{0} \geq 0$, and $h>0$ are constants. Suppose that

(a $\left.\mathrm{a}_{1}\right)\left|f_{i}(t, s, u)\right| \leq p_{i}(t, s) h_{i}(|u|)$, where $p_{i} \in C\left(\left[t_{0}, \infty\right) \times\left[t_{0}, \infty\right), \mathbb{R}_{+}\right), h_{i} \in C\left(\mathbb{R}_{+}, \mathbb{R}_{+}\right)$is continuous and nondecreasing on $\mathbb{R}_{+}$and is positive on $(0, \infty)$ such that $h_{1} \propto h_{2}, p_{i}(t, s)$ is nondecreasing in $t$ for each fixed $s, \beta_{i} \in(0,1), \gamma_{i}>1-\frac{1}{p}$ and $\frac{1}{p}+\beta_{i}+\gamma_{i}-2 \geq 0(p>1$, $i=1,2)$; 
$\left(\mathrm{a}_{2}\right) \alpha, \beta \in C^{1}\left(\left[t_{0}, \infty\right), \mathbb{R}_{+}\right), \alpha(t)$ is a nondecreasing function, $\beta(t) \leq t, \alpha(t) \leq t$, and $0<$ $\alpha(t)-\beta(t) \leq h$ for $t \geq t_{0}$;

$\left(\mathrm{a}_{3}\right) a(t)$ is continuous $\left[t_{0}, \infty\right)$.

First of all, we give an estimate for the solutions of (3.14).

Corollary 3.3 Suppose that $\left(\mathrm{a}_{1}\right)-\left(\mathrm{a}_{3}\right)$ hold, and $\max _{s \in\left[\alpha\left(t_{0}\right)-h, t_{0}\right]}|\psi(s)| \leq 3^{1-1 / q}\left|a\left(t_{0}\right)\right|$. Then any solution $x(t)$ of (3.14) has the estimate

$$
u(t) \leq\left(Q_{2}^{-1}\left(Q_{2}\left(\eta_{2}(t)\right)+3^{q-1} c_{2}^{q}(t) \int_{t_{0}}^{t} p_{2}^{q}(t, s) d s\right)\right)^{\frac{1}{q}}
$$

for $t_{0} \leq t \leq t^{*}$, where

$$
\eta_{2}(t):=Q_{1}^{-1}\left(Q_{1}\left(3^{q-1}\left(\max _{\tau \in\left[t_{0}, t\right]}|a(\tau)|\right)^{q}\right)+3^{q-1} c_{1}^{q}(t) \int_{t_{0}}^{t} p_{1}^{q}(t, s) d s\right),
$$

$c_{i}(t)=t^{1 / p+\alpha_{i}+\beta_{i}-2} B\left(p\left(\gamma_{i}-1\right)+1, p\left(\beta_{i}-1\right)+1\right)^{\frac{1}{p}}, \frac{1}{p}+\frac{1}{q}=1, Q_{i}^{-1}$ is the inverse of the function

$$
Q_{i}(u):=\int_{u_{i}}^{u} \frac{d s}{h_{i}^{q}\left(s^{\frac{1}{q}}\right)}, \quad u \geq u_{i}>0, i=1,2,
$$

and $t^{*}$ is the largest number such that

$$
\begin{aligned}
& Q_{1}\left(3^{q-1}\left(\max _{\tau \in\left[t_{0}, t^{*}\right]}|a(\tau)|\right)^{q}\right)+3^{q-1} c_{1}^{q}\left(t^{*}\right) \int_{t_{0}}^{t^{*}} p_{1}^{q}\left(t^{*}, s\right) d s \leq \int_{u_{1}}^{\infty} \frac{d s}{h_{1}^{q}\left(s^{\frac{1}{q}}\right)}, \\
& Q_{2}\left(\eta_{2}\left(t^{*}\right)\right)+3^{q-1} c_{2}^{q}\left(t^{*}\right) \int_{t_{0}}^{t^{*}} p_{2}^{q}\left(t^{*}, s\right) d s \leq \int_{u_{2}}^{\infty} \frac{d s}{h_{2}^{q}\left(s^{\frac{1}{q}}\right)} .
\end{aligned}
$$

Proof Let $\tilde{a}(t):=\max _{\tau \in[0, t]}\{|a(\tau)|\}$. Then $\tilde{a}(t)$ is a continuous and nondecreasing function on $\left[t_{0}, \infty\right)$. From $(3.14)$ and condition $\left(\mathrm{a}_{1}\right)$ we obtain

$$
\begin{aligned}
|x(t)| \leq & \tilde{a}(t)+\int_{t_{0}}^{t}(t-s)^{\beta_{1}-1} s^{\gamma_{1}-1} p_{1}(t, s) h_{1}(|x(s)|) d s \\
& +\int_{t_{0}}^{t}(t-s)^{\beta_{2}-1} s^{\gamma_{2}-1} p_{2}(t, s) h_{2}\left(\left|\max _{\xi \in[\beta(s), \alpha(s)]} x(\xi)\right|\right) d s \\
\leq & \tilde{a}(t)+\int_{t_{0}}^{t}(t-s)^{\beta_{1}-1} s^{\gamma_{1}-1} p_{1}(t, s) h_{1}(|x(s)|) d s \\
& +\int_{t_{0}}^{t}(t-s)^{\beta_{2}-1} s^{\gamma_{2}-1} p_{2}(t, s) h_{2}\left(\max _{\xi \in[\beta(s), \alpha(s)]}|x(\xi)|\right) d s
\end{aligned}
$$

for all $t \geq t_{0}$. Let $u(t)=|x(t)|$ for $t \in\left[\alpha\left(t_{0}\right)-h, \infty\right)$. Then

$$
\begin{aligned}
u(t) \leq & \tilde{a}(t)+\int_{t_{0}}^{t}(t-s)^{\beta_{1}-1} s^{\gamma_{1}-1} p_{1}(t, s) h_{1}(u(s)) d s \\
& +\int_{t_{0}}^{t}(t-s)^{\beta_{2}-1} s^{\gamma_{2}-1} p_{2}(t, s) h_{2}\left(\max _{\xi \in[\beta(s), \alpha(s)]} u(\xi)\right) d s, \quad t \geq t_{0}, \\
u(t)= & \left|\psi_{2}(t)\right|, \quad t \in\left[\alpha\left(t_{0}\right)-h, t_{0}\right] .
\end{aligned}
$$


Using the inequality $\max _{\xi \in[\beta(s), \alpha(s)]} u(\xi) \leq \max _{\xi \in[\alpha(s)-h, \alpha(s)]} u(\xi)$, which follows from condition $\left(\mathrm{a}_{2}\right)$, we obtain

$$
\begin{aligned}
u(t) \leq & \tilde{a}(t)+\int_{t_{0}}^{t}(t-s)^{\beta_{1}-1} s^{\gamma_{1}-1} p_{1}(t, s) h_{1}(u(s)) d s \\
& +\int_{t_{0}}^{t}(t-s)^{\beta_{2}-1} s^{\gamma_{2}-1} p_{2}(t, s) h_{2}\left(\max _{\xi \in[\alpha(s)-h, \alpha(s)]} u(\xi)\right) d s, \quad t \geq t_{0}, \\
u(t)= & \left|\psi_{2}(t)\right|, \quad t \in\left[\alpha\left(t_{0}\right)-h, t_{0}\right] .
\end{aligned}
$$

Notice that $\max _{s \in\left[\alpha\left(t_{0}\right)-h, t_{0}\right]}\left|\psi_{2}(s)\right| \leq 3^{1-1 / q}\left(\tilde{a}\left(t_{0}\right)\right)$ because $\max _{s \in\left[\alpha\left(t_{0}\right)-h, t_{0}\right]}\left|\psi_{2}(s)\right| \leq 3^{1-1 / q} \times$ $\left|a\left(t_{0}\right)\right|=3^{1-1 / q}\left(\tilde{a}\left(t_{0}\right)\right)$. From (3.18) and Theorem 2.1, we obtain (3.15). This completes the proof.

\section{Competing interests}

The author declares to have no competing interests.

\section{Acknowledgements}

This work was supported by the National Natural Science Foundation of China (No. 11461058) and Scientific Research Fund of SiChuan Provincial Education Department (No. 14ZA0296)

Received: 29 May 2015 Accepted: 26 October 2015 Published online: 25 November 2015

\section{References}

1. Gronwall, TH: Note on the derivatives with respect to a parameter of the solutions of a system of differential equations. Ann. Math. 20, 292-296 (1919)

2. Bellman, R: The stability of solutions of linear differential equations. Duke Math. J. 10, 643-647 (1943)

3. Bainov, D, Hristova, S: Differential Equations with Maxima. CRC Press, New York (2011)

4. Pachpatte, BG: Inequalities for Differential and Integral Equations. Academic Press, New York (1998)

5. Zhang, W, Deng, S: Projected Gronwall-Bellman's inequality for integrable functions. Math. Comput. Model. 34, 393-402 (2001)

6. Bihari, I: A generalization of a lemma of Bellman and its application to uniqueness problems of differential equations. Acta Math. Hung. 7, 81-94 (1956)

7. Lipovan, O: A retarded Gronwall-like inequality and its applications. J. Math. Anal. Appl. 252, $389-401$ (2000)

8. Agarwal, RP, Deng, S, Zhang, W: Generalization of a retarded Gronwall-like inequality and its applications. Appl. Math. Comput. 165, 599-612(2005)

9. Pinto, M: Integral inequalities of Bihari-type and applications. Funkc. Ekvacioj 33, 387-403 (1990)

10. Lipovan, O: A retarded integral inequality and its applications. J. Math. Anal. Appl. 285, 436-443 (2003)

11. $\mathrm{Ma}, \mathrm{QH}$, Yang, EH: Some new nonlinear delay integral inequalities. J. Math. Anal. Appl. 252, 864-878 (2000)

12. Meng, FW, Li, WN: On some new integral inequalities and their applications. Appl. Math. Comput. 148, 381-392 (2004)

13. Pachpatte, BG: On some new inequalities related to certain inequalities in the theory of differential equations. J. Math. Anal. Appl. 189, 128-144 (1995)

14. Cheung, WS: Some new nonlinear inequalities and applications to boundary value problems. Nonlinear Anal. 64, 2112-2128 (2006)

15. Kim, Y: Gronwall, Bellman and Pachpatte type integral inequalities with applications. Nonlinear Anal. 71, 2641-2656 (2009)

16. Wang, WS: A generalized retarded Gronwall-like inequality in two variables and applications to BVP. Appl. Math Comput. 191, 144-154 (2007)

17. McKee, S: The analysis of a variable step, variable coefficient linear multistep method for solving a singular integro-differential equation arising from the diffusion of discrete particles in a turbulent fluid. J. Inst. Math. Appl. 23, 373-388 (1979)

18. Henry, D: Geometric Theory of Semilinear Parabolic Equations. Lecture Notes in Mathematics, vol. 840. Springer, Berlin (1981)

19. Medved, M: A new approach to an analysis of Henry type integral inequalities and their Bihari type versions. J. Math. Anal. Appl. 214, 349-366 (1997)

20. $\mathrm{Ma}, \mathrm{QH}$, Yang, EH: Estimations on solutions of some weakly singular Volterra integral inequalities. Acta Math. Appl. Sin. 25, 505-515 (2002)

21. Ma, QH, Pečairé, J: Some new explicit bounds for weakly singular integral inequalities with applications to fractional differential and integral equations. J. Math. Anal. Appl. 341, 894-905 (2008)

22. Ye, H, Gao, J: Henry-Gronwall type retarded integral inequalities and their applications to fractional differential equations with delay. Appl. Math. Comput. 218, 4152-4160 (2011)

23. Golev, A, Hristova, S, Rahnev, A: An algorithm for approximate solving of differential equations with maxima. Comput. Math. Appl. 60, 2771-2778 (2010)

24. Angelov, V, Bainov, D: On the functional differential equations with maximums. Appl. Anal. 16, 187-194 (1983)

25. Popov, E: Automatic Regulation and Control. Nauka, Moscow (1966) 
26. Hristova, S, Stefanova, K: Linear integral inequalities involving maxima of the unknown scalar functions. Funkc. Ekvacioj 53, 381-394 (2010)

27. Henderson, J, Hristova, S: Nonlinear integral inequalities involving maxima of unknown scalar functions. Math. Comput. Model. 53, 871-882 (2011)

28. Bohner, M, Hristova, S, Stefanova, K: Nonlinear integral inequalities involving maxima of the unknown scalar functions. Math. Inequal. Appl. 12, 811-825 (2012)

29. Yan, Y: Nonlinear Gronwall-Bellman type integral inequalities with maxima. Math. Inequal. Appl. 16, 911-928 (2013)

30. Thiramanus, $P$, Tariboon, J, Ntouyas, S: Henry-Gronwall integral inequalities with maxima and their applications to fractional differential equations. Abstr. Appl. Anal. 2014, Article ID 276316 (2014)

Submit your manuscript to a SpringerOpen ${ }^{\circ}$ journal and benefit from:

- Convenient online submission

Rigorous peer review

- Immediate publication on acceptance

- Open access: articles freely available online

- High visibility within the field

- Retaining the copyright to your article 